\title{
Band-Limited Morphometric Analysis of the Intracardiac Signal: Implications for Antitachycardia Devices
}

\author{
MILTON M. MORRIS, JANICE M. JENKINS, and LORENZO A. DICARLO \\ From the Department of Electrical Engineering and Computer Science, The University of Michigan, \\ Ann Arbor, Michigan
}

\begin{abstract}
MORRIS, M.M., ET AL.: Band-Limited Morphometric Analysis of the Intracardiac Signal: Implications for Antitachycardia Devices. Inappropriate electrical therapy and power efficiency play a major role in algorithm implementation for antitachycardia devices (ATD) that capture, store, and analyze the patient electrogram as an adjunct to rate determination. Morphologically based algorithms have been demonstrated to improve specificity, thereby decreasing occurrences of inappropriate electrical therapy. However, morphologically based algorithms are power demanding. Optimization of power efficiency can be achieved by eliminating unnecessary algorithmic computation, but must not compromise the effectiveness of algorithms, which perform direct analysis on raw signals. Significant reductions can be achieved by reduced sampling rates, which allow for increased overall ATD efficiency via concomitant decreases in computation and data storage. This investigation determined the upper and lower bounds for filter cutoff frequency beyond which detection precision by an established morphometric method for arrhythmia classification, correlation waveform analysis (CWA), was unfavorable. Four measurement statistics were used. In ten patients with inducible VT and VF, all bipolar intraventricular electrograms were classified correctly with a minimum passband of 10-50 Hz using any of the four measurement statistics. There was $\geq 80 \%$ correct classification using all four measurement statistics with passbands having low frequency cutoffs $\leq 15 \mathrm{~Hz}$ and high frequency cutoffs $\geq 50 \mathrm{~Hz}$. Correct classification of $\geq 90 \%$ of unipolar electrograms during NSR, VT, and VF occurred using all four measurement statistics with a passband of 1-50 $\mathrm{Hz}$. There was $\geq 80 \%$ correct classification with passbands 1, 10, 15, or 20-500 Hz and 10-50 Hz. The classification of NSR, VT, and VF was most accurate on an intrapatient basis. Accuracy decreased using an interpatient rhythm classification. Optimum filter settings of 1-50 Hz and 10-50 Hz were determined for unipolar and bipolar electrograms, respectively. Sampling data at $120 \mathrm{~Hz}$ was found to be sufficient. Bipolar electrode configuration statistically outperfomed unipolar data. In conclusion, morphometric analysis of bipolar and unipolar intraventricular electrograms appears to be achievable using band limited data and reduced sampling rates. (PACE 1997; 20[Pt. I]:34-42)
\end{abstract}

\section{intracardiac electrogram, filtering, bioelectric signal, ventricular tachycardia, ventricular fibrillation, antitachycardia device}

\section{Introduction}

The search for better arrhythmia classification methods has been an important component in the

This work was partially supported by NSF grants EID-9018746 and GER-9023514, NIH grant 1 F31 GM17240-01, and by Intel ${ }^{\circledR}$ Corp.

Address for reprints: Milton M. Morris, Medical Computing Laboratory, 4421 EECS Bldg., Ann Arbor, MI 48109-2122. Fax: (313) 763-8041.

Received March 20, 1995; revision July 10, 1995; accepted September 28, 1995. ongoing design and development of implantable antitachycardia devices (ATDs). Much of this work parallels advances made years earlier in computerized electrocardiography and coronary intensive care monitoring. Computer systems for these applications have achieved a high level of sophistication in analysis and classification of arrhythmias. Similar techniques have been developed for ATDs, but with the need to address inappropriate electrical therapy, computational constraints, as well as signal sampling and storage limitations imposed by small battery operated devices, new and 


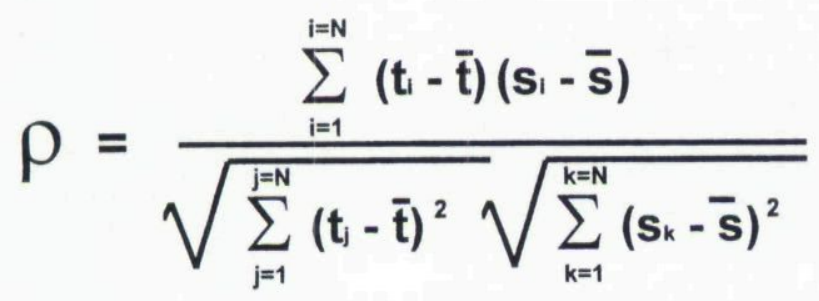

Figure 1. Correlation waveform analysis equation. $\rho=$ normalized correlation coefficient, $t_{i}, t_{j}=$ ith and $j$ th points of the template sequence, $s_{i}, s_{k}=i$ th and $k t h$ point in signal sequence, $\bar{t}, \bar{s}=$ mean value of the template and signal sequences, $N=$ window length (length of data sequence).

imaginative solutions must be derived.

Inappropriate electrical therapy from currently available commercial and investigational devices that use rate analysis have been reported during documented periods of normal sinus rhythm (NSR), sinus tachycardia, and supraventricular tachycardias (SVT) including atrial flutter $(\mathrm{AFl})$ and atrial fibrillation (AF). The reported incidence has ranged from $10 \%-41 \% .{ }^{1-5}$ Inappropriate therapy has decreased in incidence, but persists despite incorporation of increasingly complex forms of rate analysis into ATDs.

Morphological assessment of the intracardiac electrogram has emerged as a promising technique for more specific assessment of abnormal cardiac conduction. ${ }^{6-25} \mathrm{~A}$ variety of new morphological methods have been advanced in investigative reports using both time- and frequency-domain analysis. Correlation waveform analysis (CWA), a proposed time-domain template matching algorithm, ${ }^{10,25-27}$ has been applied to the detection of VT from the intraventricular electrogram (IVE), ${ }^{13}$ recognition of retrograde atrial activation in intraatrial electrogram (IAE) analysis, ${ }^{21}$ classification of VTs of different morphologies in IVEs, ${ }^{28}$ and separation of VT from ventricular fibrillation (VF) in IVEs. ${ }^{29}$

The CWA metric (Fig. 1) is a mathematical measure of likeness between a template and a signal for comparison. The correlation coefficient generated by this equation is normalized to take values between \pm 1 .

The performance of CWA in classification of abnormal cardiac activation in intracardiac elec- trograms has duplicated its long-term success and acceptance in surface ECG, positioning CWA as a standard by which the performances of other, more computationally efficient morphologically based algorithms can be measured.

In considering digital signal analysis of the electrogram, a minimum effective passband with acceptable sampling rates is important in the implementation of these algorithms. A diminished sampling rate has a significant impact on the power requirements for both storage and computational analysis of electrograms.

Bandpass filtering of electrograms is used as a first stage in ATDs for elimination of baseline wander and enhancement of higher frequency components contained in the depolarization for triggering purposes. Unfortunately, filtering of the electrogram invariably results in distortion and

\section{Ventricular Tachycardia $(1-500 \mathrm{~Hz})$}

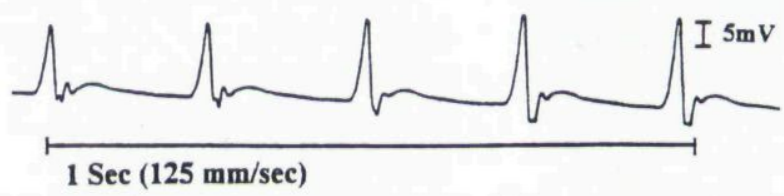

Ventricular Tachycardia $(10-25 \mathrm{~Hz})$

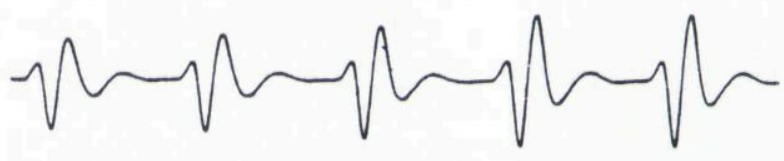

Ventricular Fibrillation (1-500Hz)

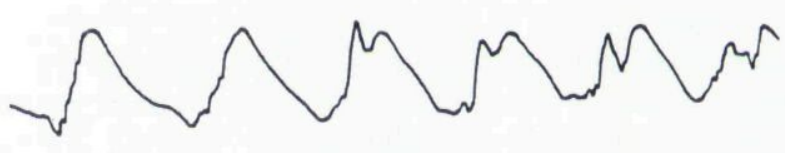

Ventricular Fibrillation $(\mathbf{1 0 - 2 5 H z})$

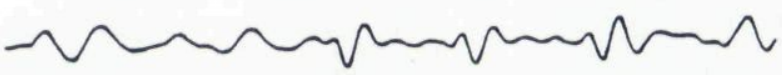

Figure 2. Effect of filtering on intracardiac waveforms. This figure shows an example of VT at 1-500 Hz and $10-25 \mathrm{~Hz}$ (top two tracings) and VF in the same patient at $1-500 \mathrm{~Hz}$ and $10-25 \mathrm{~Hz}$ (lower two tracings). Identical scaling is used for each passage. 
loss of signal information, which might impact upon morphologically based algorithms. Figure 2 illustrates the effect of analog bandpass filtering on VT and VF in the same patient.

The determination of minimum effectual passband was sought in order to define a suitable sampling rate for signal processing by microprocessor based algorithms. The primary purpose of this study was to determine the reasonable upper and lower limits of bandpass filtering of the ventricular intracardiac electrogram that would allow morphometric separation of abnormal and normal conduction for classification of rhythms. The results would provide a definitive guide to allowable bandpass ranges.

\section{Methods and Materials}

The method used for comparative analysis was the morphological metric CWA. Four variables affecting arrythmia detection by ATDs analyzed in this study were electrode configuration, signal frequency content, sampling rate, and patient classification. Other potential variables (i.e., method of triggering, preamplification, etc.) were held constant. Intraventricular electrograms analyzed in this study were recorded from the right ventricular apex (1-500 Hz) during clinical cardiac electrophysiology studies of patients undergoing clinical evaluation and treatment. Written informed consent was obtained from each patient. Three 6 Fr quadropolar electrode catheters (USCI Division, C.R. Bard Inc., Billerica, MA, USA) with an interelectrode distance of $1 \mathrm{~cm}$ were introduced and advanced under fluoroscopic guidance. Each electrode catheter had three platinum ring electrodes that were cylinders $2 \mathrm{~mm}$ in diameter and $2 \mathrm{~mm}$ in length. The platinum tip electrode of each catheter consisted of a half-sphere of $2 \mathrm{~mm}$ in diameter attached to a cylinder of $2 \mathrm{~mm}$ in diameter and $1 \mathrm{~mm}$ in length. The resultant surface area of each ring and tip electrode was 12.6 $\mathrm{mm}^{2}$. One electrode catheter was positioned in the high right atrium or right atrial appendage. Two electrode catheters were positioned in the right ventricular apex for right ventricular apex pacing and recording. Episodes of induced monomorphic VT and VF having a duration of $\geq 9$ seconds were recorded from bipolar and unipolar electrodes located in the right ventricular apex. ${ }^{13}$ Intraventric- ular electrograms were recorded continuously on FM magnetic tape at a tape speed of 3.75 in (9.5 $\mathrm{cm}) / \mathrm{sec}$ (bandwidth of 0-500 Hz) (HewlettPackard ${ }^{\circledR}$ Model 3968A, San Diego, CA, USA) after signal amplification and filtering at $1250 \mathrm{~Hz}$. Amplifier gain and filter settings were held constant during the entire recording procedure, and all eletrogram amplitudes were computed using a $1-\mathrm{mV}$ calibration signal entered as a reference at the time of recording. Each recording was assigned a case number and catalogued for subsequent off-line retrieval (Ann Arbor Electrogram Libraries, Ann Arbor, MI, USA). Ten patients with diagnosed and documented episodes of NSR, and both VT and VF were recorded using bipolar and unipolar electrodes. The bipolar and unipolar electrograms were filtered at 16 distinct settings using high pass filtering at $1,10,15$, and $20 \mathrm{~Hz}$, and low pass filtering at 500, 100, 50, and $25 \mathrm{~Hz}$. Data were digitized with a commercial analog-todigital (A/D) acquisition system (DATAQ ${ }^{\circledR}$, Akron, OH, USA), at sampling rates shown in Table I, in accordance with the Nyquist criterion. The conditioned signals were then processed on a cycle-by-cycle basis for arrhythmia classification using CWA.

Table I.

Filter Bandwidths

\begin{tabular}{cc} 
Bandwidth $(\mathbf{H z})$ & Sampling Freq. $\mathbf{( H z})$ \\
\hline $1-500$ & 1000 \\
$1-100$ & 250 \\
$1-50$ & 120 \\
$1-25$ & 70 \\
$10-500$ & 1000 \\
$10-100$ & 250 \\
$10-50$ & 120 \\
$10-25$ & 70 \\
$15-500$ & 1000 \\
$15-100$ & 250 \\
$15-50$ & 120 \\
$15-25$ & 70 \\
$20-500$ & 1000 \\
$20-100$ & 250 \\
$20-50$ & 120 \\
$20-25$ & 70 \\
\hline
\end{tabular}


Analog data were filtered using a digitally tuned variable Krohn-Hite filter (Series 3320 Model 3323, Krohn-Hite Corporation, Avon, MA, USA). This series and model is tunable over the range of $0.01 \mathrm{~Hz}$ to $99.9 \mathrm{kHz}$. The passband gain was unity $(0 \mathrm{~dB})$, with attenuation rates of $24 \mathrm{~dB}$ per octave outside the passband. Response characteristics were that of a four-pole, maximally flat Butterworth. The attenuation slope is nominally $24 \mathrm{~dB}$ per octave per channel in low pass or high pass mode.

\section{Computer Processing}

Individual complexes (depolarizations) were recognized using an autoadjusting software trigger. Subsequent verification of trigger locations was performed with the assistance of a cardiologist. A template of a normal ventricular electrogram for each patient was obtained by signal averaging ventricular depolarization waveforms from a passage of NSR to produce a representative waveform. A minimum of 16 depolarizations were used, based on the knowledge that signal averaging reduces the signal-to-noise $(\mathrm{S} / \mathrm{N})$ ratio by a factor of $n^{1 / 2}$, where $n$ is the number of signals averaged. ${ }^{30}$ Separate passages of NSR, VT, and VF were then processed by CWA on a cycle-by-cycle basis using the NSR template as reference.

\section{Statistics Applied for Analysis}

Key statistics were generated using the cycleby-cycle correlation coefficients (CCs) of each passage. Each statistic was used individually as a method for intrapatient separation and combined statistics were examined for interpatient separation. The statistical metrics are described below.

Delta-CC was defined as the absolute difference between upper and lower bound in the range of CCs produced by CWA for a passage containing multiple cycles. Because CC represents the normalized correlation coefficient yielding a value between \pm 1 , Delta-CC yields a number between 0 and 2. The limitation of using Delta-CC as a discriminatory method was the magnitude of the separation factor (margin) between different rhythms.

Standard deviation of the correlation coefficients was calculated and examined as a candidate for separation, particularly the separation of VT and VF. ${ }^{29}$ Limitations of using standard devia- tion as a discriminatory method were similar to Delta-CC.

Area, defined as the product of Delta-CC and standard deviation, was used as a measure of consistency of the CC values. Since the area was derived by multiplication of two statistics, the resultant value exaggerated the consistency (or inconsistency) and produced greater separation of distinct rhythms.

Volume, defined as the product between Area and the inverse of the minimum absolute value of CCs, had all of the qualities of the area statistic with more separation space (i.e., larger statistical margin).

These statistics were the product of a pilot study the author conducted, which analyzed correlation coefficients in conjunction with the rhythms that produced them. It was determined that statistics that measure consistency (or inconsistency) would best classify the three rhythms of this study.

Intrapatient and interpatient thresholds were used for separation of NSR from VT, and VT from VF. At all filter settings that gave successful performance by CWA, there was no statistical overlap between NSR and VF.

\section{Delta-CC vs. Volume: Bipolar $10-50 \mathrm{~Hz}$}

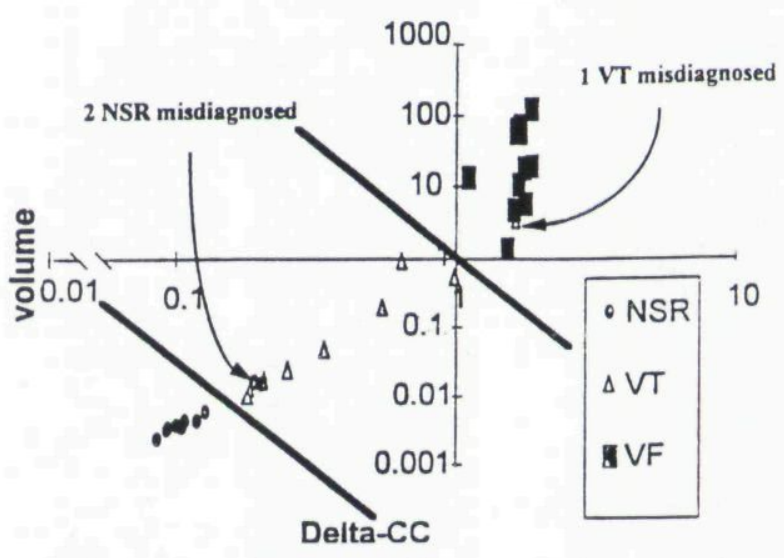

Figure 3. Two-dimensional plot (Delta-CC vs volume, log-log scale) of bipolar data filtered at 10-50 Hz. Bold lines represent decision boundaries between NSR, VT, and VF for two-feature analysis (Delta-CC versus volume). Two NSR passages were misdiagnosed as VT, and one VT was misdiagnosed as VF. 


\section{Performance Rating}

Intrapatient

The quality of performance by CWA was based on the percentage of patients whose rhythms were correctly classified using the key statistics described above (Delta-CC, standard deviation, area, and volume). Correct classification is assessed with the use of decision boundaries.

\section{Interpatient}

Specificity and sensitivity were used to describe CWA interpatient (group-specific) performance. Combinations of the four key statistics were used to form two-dimensional plots, and correct classification was assessed using a linear decision criterion. Figure 3 shows two linear boundaries separating a cluster of NSR from V'T and VT from VF.

Because of the severe consequences of a failure of detection in practical use, and for the purpose of this study, optimal specificity was determined with sensitivity $=1$.

\section{Results}

Results are given both for intrapatient (patient-specific) classification and interpatient (group-specific) classification. For intrapatient classification, each patient had two patient-specific thresholds (one separating NSR from VT and one separating VT from VF) that varied from patient to patient. For interpatient classification, two thresholds distinguishing the three rhythms of interest were globally set and applied to all patient data. The results are reported separately for unipolar and bipolar electrode configurations.

\section{Intrapatient (Patient-Specific) Analysis}

\section{Bipolar}

The optimal filter setting for bipolar data analyzed by CWA was $10-50 \mathrm{~Hz}$ (Table II). No statistical overlap occurred in the four methods used for rhythm classification, and the corresponding sampling rate was reduced to $120 \mathrm{~Hz}$ (relative to a wide band filter sampling rate of $1,000 \mathrm{~Hz}$ ). The four statistical metrics were most consistent

Table II.

Bipolar Results

\begin{tabular}{|c|c|c|c|c|c|c|c|c|}
\hline \multirow[b]{2}{*}{ Setting $(\mathbf{H z})$} & \multicolumn{2}{|c|}{ Delta-CC } & \multicolumn{2}{|c|}{ Stan. Dev. } & \multicolumn{2}{|c|}{ Area } & \multicolumn{2}{|c|}{ Volume } \\
\hline & NSR-VT & VT-VR & NSR-VT & VT-VF & NSR-VT & VT-VF & NSR-VT & VT-VF \\
\hline $1-500$ & 100 & 90 & 100 & 80 & 100 & 90 & 100 & 90 \\
\hline $1-100$ & 100 & 100 & 100 & 80 & 100 & 90 & 100 & 90 \\
\hline $1-50$ & 90 & 100 & 90 & 90 & 90 & 90 & 90 & 100 \\
\hline $1-25$ & 100 & 100 & 90 & 100 & 100 & 100 & 100 & 100 \\
\hline $10-500$ & 100 & 90 & 100 & 90 & 100 & 90 & 100 & 80 \\
\hline $10-100$ & 90 & 100 & 90 & 90 & 80 & 90 & 90 & 80 \\
\hline $10-50$ & 100 & 100 & 100 & 100 & 100 & 100 & 100 & 100 \\
\hline $10-25$ & 90 & 90 & 90 & 90 & 90 & 90 & 90 & 90 \\
\hline $15-500$ & 90 & 80 & 90 & 90 & 90 & 90 & 100 & 80 \\
\hline $15-100$ & 100 & 90 & 90 & 90 & 100 & 90 & 100 & 90 \\
\hline $15-50$ & 80 & 80 & 80 & 90 & 80 & 90 & 80 & 80 \\
\hline $15-25$ & 90 & 50 & 90 & 70 & 90 & 70 & 90 & 90 \\
\hline $20-500$ & 100 & 70 & 100 & 70 & 100 & 70 & 100 & 80 \\
\hline $20-100$ & 80 & 100 & 90 & 100 & 80 & 100 & 80 & 90 \\
\hline $20-50$ & 90 & 50 & 90 & 70 & 90 & 80 & 90 & 60 \\
\hline $20-25$ & 80 & 80 & 70 & 70 & 80 & 80 & 80 & 60 \\
\hline
\end{tabular}

This table gives the percentage of correctly classified passages (10 patients) for bipolar recordings. The first column heading gives the filter setting and column headings $2-5$ give the analysis method. NSR = normal sinus rhythm; Stan. Dev. = standard deviation; VF = ventricular fibrillation; VT = ventricular tachycardia. 
Table III.

Unipolar Results

\begin{tabular}{|c|c|c|c|c|c|c|c|c|}
\hline \multirow[b]{2}{*}{ Setting (Hz) } & \multicolumn{2}{|c|}{ Delta-CC } & \multicolumn{2}{|c|}{ Stan. Dev. } & \multicolumn{2}{|c|}{ Area } & \multicolumn{2}{|c|}{ Volume } \\
\hline & NSR-VT & VT-VR & NSR-VT & VT-VF & NSR-VT & VT-VF & NSR-VT & VT-VF \\
\hline $1-500$ & 100 & 90 & 90 & 80 & 100 & 80 & 100 & 90 \\
\hline $1-100$ & 90 & 80 & 90 & 70 & 90 & 70 & 90 & 70 \\
\hline $1-50$ & 100 & 90 & 90 & 90 & 100 & 90 & 100 & 100 \\
\hline $1-25$ & 70 & 90 & 80 & 90 & 80 & 90 & 70 & 80 \\
\hline $10-500$ & 90 & 100 & 100 & 100 & 90 & 100 & 90 & 90 \\
\hline $10-100$ & 100 & 70 & 100 & 70 & 100 & 90 & 100 & 70 \\
\hline $10-50$ & 90 & 100 & 90 & 90 & 90 & 90 & 90 & 90 \\
\hline $10-25$ & 90 & 90 & 100 & 90 & 90 & 90 & 90 & 70 \\
\hline $15-500$ & 100 & 90 & 100 & 80 & 100 & 90 & 100 & 80 \\
\hline $15-100$ & 90 & 80 & 90 & 80 & 90 & 70 & 90 & 70 \\
\hline $15-50$ & 100 & 100 & 100 & 70 & 100 & 70 & 100 & 80 \\
\hline $15-25$ & 70 & 60 & 80 & 70 & 80 & 70 & 80 & 60 \\
\hline $20-500$ & 90 & 90 & 90 & 80 & 90 & 100 & 90 & 100 \\
\hline $20-100$ & 100 & 80 & 100 & 80 & 100 & 80 & 100 & 60 \\
\hline $20-50$ & 90 & 60 & 90 & 60 & 90 & 70 & 90 & 90 \\
\hline $20-25$ & 70 & 70 & 60 & 50 & 70 & 60 & 70 & 80 \\
\hline
\end{tabular}

This table gives the percentage of correctly classified passages (10 patients) for unipolar recordings. The first column heading gives the filter setting and column headings $2-5$ give the analysis method. NSR = normal sinus rhythm; Stan. Dev. = standard deviation; VF = ventricular fibrillation; $\mathrm{VT}=$ ventricular tachycardia.

across patients for NSR. The metrics lost consistency for VT and VF where VF contained the greatest variance.

Several other filter settings resulted in $\geq 80 \%$ correct classification of patient rhythms via all four statistics. Overall, CWA correctly classified $\geq$ $80 \%$ of patient rhythms for filter settings with low pass cutoff of $\geq 50 \mathrm{~Hz}$ combined with high pass cutoff of $\leq 15 \mathrm{~Hz}$.

\section{Unipolar}

The optimal filter setting for unipolar data was $1-50 \mathrm{~Hz}$ (Table III). Overall, the percentage of patients with correctly classified rhythms at the optimal unipolar filter setting using all four statistics was lower $(90 \%-100 \%)$ than bipolar data $(100 \%)$.

The high pass and low pass frequency cutoffs for correct classification of $\geq 80 \%$ of patient rhythms ranged considerably using unipolar data. When the low pass cutoff was set to $500 \mathrm{~Hz}$, correct classification was achieved in $\geq 80 \%$ of patient rhythms regardless of high pass cutoff. Overall, the $10-50 \mathrm{~Hz}$ filter setting allowed for $\geq 90 \%$ correct classification of patient rhythms. As in the bipolar case, the four statistical metrics were most consistent across patients for NSR. The metrics lost consistency for VT and VF where VF contained the greatest variance.

\section{Interpatient (Group-Specific) Analysis}

Two-dimensional charts were used for interpatient rhythm classification. One of the charts is illustrated (log-log scale) in Figure 3. The charts include the filter settings of interest, electrode configurations (bipolar or unipolar), and statistical combinations (Delta-CC vs volume, standard deviation vs volume, or area vs volume). Results presented here are for the two best performing filter settings $(1-50 \mathrm{~Hz}, 10-50 \mathrm{~Hz})$.

\section{Bipolar}

Table IV summarizes specificity with sensitivity $=1$, using three combinations of statistics. For bipolar electrograms, each of the three statistical combinations produced similar results for separation of VT and NSR. There is, however, a difference when separating VT from VF. Delta-CC versus volume at $1-50 \mathrm{~Hz}$ (bipolar) was the best 
method for maximizing specificity while maintaining a sensitivity $=1$. All metrics yielded equivalent results using a filter setting of $10-50$ $\mathrm{Hz}$.

\section{Unipolar}

For unipolar electrograms at $1-50 \mathrm{~Hz}$ and $10-50 \mathrm{~Hz}$ settings, area versus volume yielded the best results (Table IV).

\section{Performance of Statistical Methods for Bipolar and Unipolar Lead Configurations}

Referring to Tables II and III, wide band data recorded at $1-500 \mathrm{~Hz}$ provided similar statistical performance in both unipolar and bipolar lead configurations. Subsequent filtering yielded a trade-off in level of statistical performance.

\section{Intrapatient}

The most accurate statistics for distinguishing VT from NSR were volume $(94.3 \%)$ and area $(92.4 \%)$ using bipolar and unipolar lead configu- rations, respectively. The most accurate statistics for distinguishing VT from VF were Area (89.2\%) and Delta-CC (84.8\%) using bipolar and unipolar lead configuration, respectively. The largest margins of separation were found using the volume statistics.

\section{Interpatient}

A low specificity was found for VT versus VF using area versus volume for bipolar data filtered at $1-50 \mathrm{~Hz}$ (Table IV). However, area versus volume at $10-50 \mathrm{~Hz}$ performed as well as Delta-CC versus volume for bipolar data. In contrast, standard deviation versus volume was exceeded by Delta-CC versus volume by a difference of 0.1 in its specificity for NSR versus VT and VT versus VF using bipolar data filtered at $1-50 \mathrm{~Hz}$ (Table IV).

In contrast, specificity was consistently and unacceptably low for unipolar data using any of the three statistical combinations for analysis

Table IV.

Specificity of Each Statistic with Sensitivity $=1$

\begin{tabular}{|c|c|c|c|c|}
\hline \multicolumn{5}{|c|}{ A } \\
\hline \multirow{2}{*}{$\begin{array}{l}\text { Delta-CC vs Volume } \\
\text { Filter Setting }(\mathrm{Hz})\end{array}$} & \multicolumn{2}{|c|}{ NSR vs VT } & \multicolumn{2}{|c|}{ VT vs VF } \\
\hline & Bipolar & Unipolar & Bipolar & Unipolar \\
\hline $1-50$ & 0.6 & 0.4 & 0.8 & 0.7 \\
\hline $10-50$ & 0.8 & 0.5 & 0.9 & 0.5 \\
\hline \multicolumn{5}{|c|}{ B } \\
\hline & \multicolumn{2}{|c|}{ NSR vs VT } & \multicolumn{2}{|c|}{ VT vs VF } \\
\hline $\begin{array}{l}\text { Stand. Dev. vs Volume } \\
\text { Filter Setting (Hz) }\end{array}$ & Bipolar & Unipolar & Bipolar & Unipolar \\
\hline $1-50$ & 0.5 & 0.3 & 0.7 & 0.8 \\
\hline $10-50$ & 0.8 & 0.5 & 0.9 & 0.5 \\
\hline \multicolumn{5}{|c|}{ C } \\
\hline & \multicolumn{2}{|c|}{ NSR vs VT } & \multicolumn{2}{|c|}{ VT vs VF } \\
\hline $\begin{array}{l}\text { Area vs Volume } \\
\text { Filter Setting (Hz) }\end{array}$ & Bipolar & Unipolar & Bipolar & Unipolar \\
\hline $1-50$ & 0.6 & 0.6 & 0.3 & 0.8 \\
\hline $10-50$ & 0.8 & 0.6 & 0.9 & 0.5 \\
\hline
\end{tabular}

NSR $=$ normal sinus rhythm; Stan. Dev. = standard deviation; VF = ventricular fibrillation; VT = ventricular tachycardia. 
across both filter settings (Table IV). Overall area versus volume with a filter setting of $1-50 \mathrm{~Hz}$ or $10-50 \mathrm{~Hz}$ yielded best unipolar results.

\section{Discussion}

The performance of CWA in morphological separation of rhythms varied, based on electrode configuration, frequency content of the analog signal, sampling rate, and patient classification (interpatient vs intrapatient). Filter settings of $1-50$ $\mathrm{Hz}$ and $10-50 \mathrm{~Hz}$ sampled at $120 \mathrm{~Hz}$ provided the most effective intrapatient and interpatient rhythm separation for unipolar and bipolar electrodes, respectively. This designation is based on a combination of performance versus effective sampling rate.

The best classification was found using an intrapatient (patient-specific) discriminant compared to an interpatient (group-specific) basis. This should not pose a problem in implementation since, in present devices, zones for tachycardia detection are patient-specific and programmable. Patient-specific adjustment of discriminant boundaries for morphological analysis could be expected to be similarly selected in the future.

\section{Bipolar Versus Unipolar Electrogram Morphology}

Results differed for unipolar and bipolar data. This is not surprising, since the two lead configurations create morphologically different signals. Bipolar electrograms provide near-field information (i.e., proximal view of electrical activity). Unipolar electrograms represent far-field information (i.e., global view of electrical activity).

\section{Computational Considerations}

Because of its consistently reliable performance, CWA with a sampling rate of $1,000 \mathrm{~Hz}$ has

\section{References}

1. Echt DS, Armstrong K, Schmidt P, et al. Clinical experience, complications and survival in 70 patients with automatic implantable cardioverter/ defibrillator. Circulation 1985; 71:289-296.

2. Steinberg JS, Sugalski JS, Haratonic K, et al. Car- been used as a performance measure for morphometric analysis in our laboratory. The results of the present study demonstrate that filter settings of $1-50 \mathrm{~Hz}$ (unipolar) and $10-50 \mathrm{~Hz}$ (bipolar) provide similar performance by CWA as to performance at wider passbands $(1-500,100 \mathrm{~Hz})$. Considerable computational savings are achieved by the concomitant reduced sampling rate associated with these filter settings. This reduction of sample points decreases the number of multiplications, additions, and subtractions by as much as a factor of ten. Additional savings in memory allocation for template storage and buffered data storage have the potential of further power reduction in implantable devices.

\section{Triggering}

Because the CWA morphometric is highly trigger dependent, precise alignment of cardiac waveforms is critical to subsequent analysis performance ${ }^{30}$ In the present study, the impact of trigger misalignment increased as sampling rate was reduced, providing fewer data points per cycle for trigger activation. This loss of precision was pronounced at sampling rates $<120 \mathrm{~Hz}$ and contributed to lower classification success.

\section{Conclusion}

The findings of this study demonstrate that morphometric analysis by ATDs is feasible on an intrapatient basis using a unipolar filter setting of $1-50 \mathrm{~Hz}$ and a bipolar filter setting of $10-50 \mathrm{~Hz}$ with a sampling rate of $120 \mathrm{~Hz}$. Narrow bandwidth filtering and a reduced sampling rate of $120 \mathrm{~Hz}$ will decrease computational requirements and increase the number of electrograms available for data storage (memory) in digital devices without significantly impairing the diagnostic accuracy of time-domain analysis.

diac rhythm precipitating automatic implantable defibrillator discharges in outpatients: Observations from transtelephonic recordings. (abstract) Circulation 1989; 80:530.

3. Gabry MD, Brodman R, Johnston D, et al. Auto- 
matic implantable cardioverter-defibrillator: $\mathrm{Pa}$ tient longevity and shock delivery analysis. J Am Coll Cardiol 1987; 9:1349-1356.

4. Grimm W, Flores BF, Marchlinski FE. Electrocardiographically documented unnecessary, spontaneous shocks in 241 patients with implantable cardioverter defibrillators. PACE 1992; 15(Pt. I):1667-1673.

5. Sandler MJ, Kutalek SP. Inappropriate discharge by an implantable cardioverter defibrillator: Recognition of myopotential sensing using telemetered intracardiac electrograms. PACE 1994; 17(Pt. I):665-671.

6. Stephany G, Ropella K. Real-time estimation of a Magnitude-square coherence for use in implantable devices. New York, NY, IEEE Press, 1992, pp. 375-378.

7. Langberg JL, Gibbs WJ, Auslander DM, et al. Identification of ventricular tachycardia with use of the morphology of the endocardial electrogram. Circulation 1988; 77:1363-1369.

8. Throne RD. Analysis of Template Matching Methods for Intracardiac Electrogram Analysis. Ph.D. Thesis, University of Michigan, 1990.

9. Chiang CM. Real-Time Automatic Detection of Cardiac Arrhythmias By Rate and Morphology Analysis of Intracardiac Electrograms. Ph.D. Thesis, University of Michigan, 1993.

10. Jenkins JM, DiCarlo LA, Chang CM. Impact of filtering upon ventricular tachycardia identification by correlation waveform analysis. PACE 1991; 14:1809.

11. Santel D, Mehra R, Olson W, et al. Integrative algorithm for detection of ventricular tachyarrythmias from the intracardiac electrogram. New York, NY, IEEE Press, 1986, pp. 175-177.

12. Throne RD, Jenkins JM, DiCarlo LA. A comparison of four new time-domain techniques for discriminating monomorphic ventricular tachycardia from sinus rhythm using ventricular waveform morphology. IEEE Trans Biomed Eng 1991; 38: 561-570.

13. Lin D, DiCarlo LA, and Jenkins JM. Identification of ventricular tachycardia using intracavity ventricular electrograms: Analysis of time and frequency domain patterns. PACE 1988; 11: 1592-1606

14. Throne RD, Jenkins JM, Winston SA, et al. Paroxysmal bundle branch block of supraventricular origin: A possible source of misdiagnosis in detecting ventricular tachycardia using ventricular electrogram morphology. PACE 1990;13: 453-458.

15. Lin D, DiCarlo LA, Jenkins JM. Identification of ventricular tachycardia using morphology analysis of the intraventricular electrogram. (abstract) J Electrocardiol 1988; 21(Suppl):120.

16. DiCarlo LA, Lin D, Jenkins JM. Analysis of time and frequency domain patterns to distinguish ventricular tachycardia from sinus rhythm using en- docardial electrograms. (abstract) Circulation 1987; 76:280.

17. Lin D, Jenkins JM, Jadvar H, et al. Analysis of time and frequency domain patterns of endocardial electrograms to distinguish ventricular tachycardia from sinus rhythm. New York, NY, IEEE Press, 1986, pp. 171-174.

18. Throne RD, Jenkins JM, Winston SA, et al. Derivative area method: A new technique for detecting ventricular tachycardia. (abstract) Circulation 1989; 80:II-658.

19. Throne RD, Jenkins JM, Winston SA, et al. The bin area method: A new computationally efficient algorithm for intracardiac electrogram analysis. (abstract) J Am Coll Cardiol 1990; 17.

20. Throne RD, Jenkins JM, Winston SA, et al. Ventricular tachycardia versus supraventricular tachycardia with aberration: An analysis of "bundle branch" characteristics using intracardiac electrograms. (abstract) PACE 1989; 12:662.

21. Throne RD, Jenkins JM, Winston SA, et al. Discrimination of retrograde from anterograde atrial activation using intracardiac electrogram waveform analysis. PACE 1989; 12:1622-1630.

22. Finelli CJ, Li P, Jenkins JM, et al. Intraventricular electrogram morphology: Effect of increased heart rate with and without accompanying changes in sympathetic tone. New York, NY, IEEE Press, 1990, pp. 115-118.

23. Jenkins JM, DiCarlo LA, Chiang CM. Impact of filtering upon ventricular tachycardia identification by correlation waveform analysis. PACE 1991; 14: 1809-1814.

24. Throne RD, Jenkins JM, DiCarlo LA. The bin area method: A computationally efficient technique for analysis of ventricular and atrial intracardiac electrograms. PACE 1990; 13:1286-1297.

25. Lin D, Jenkins JM, DiCarlo LA, et al. Arrhythmia diagnosis using morphology and timing from atrial and ventricular leads. New York, NY, IEEE Press, 1988, pp. 159-162.

26. DiCarlo LA, Lin D, Jenkins JM. Automated interpretation of cardiac arrhythmias. J Electrocardiol 1993; 26:53-67.

27. Tomaselli GF, Nielsen AP, Finke WL, et al. Morphologic differences of the endocardial electrogram in beats of sinus and ventricular origin. PACE 1988; 11:254-262.

28. Throne RD, Jenkins JM, Winston SA, et al. Use of tachycardia templates for recognition of recurrent monomorphic ventricular tachycardia. New York, NY, IEEE Press, 1989, pp. 171-174.

29. DiCarlo LA, Jenkins JM, Winston SA, et al. Differentiation of ventricular tachycardia from ventricular fibrillation using intraventricular electrogram morphology. Am J Cardiol 1992; 9:820-822.

30. Webster JG. Medical Instrumentation: Application and Design. 2nd Ed. Boston, MA, Houghton Mifflin Company, 1992, pp. 332-333. 
This document is a scanned copy of a printed document. No warranty is given about the accuracy of the copy. Users should refer to the original published version of the material. 